\title{
Offline and CAD-GEANT4 software of the DAMPE mission
}

\author{
Andrii Tykhonov*广 \\ DPNC, Université de Genève, CH-1211 Genève 4, Switzerland \\ E-mail: andrii.tykhonovecern.ch
}

\begin{abstract}
An overview is given for the offline software framework of the DAMPE satellite mission. In particular, the problem of converting CAD detector model into the GEANT4-compatible format, GDML, is solved. The geometry of the DAMPE payload is implemented as GDML from the corresponding CAD drawings of the detector. The output of CAD-GDML conversion is parsed further to identify sensitive parts of the detector and convert them into simple geometries instead of tessellated ones. The GDML model is used both by the simulation and reconstruction jobs, and all other algorithms that may deal with the detector geometry. Next, preliminary results of the alignment of silicon tracker of DAMPE are presented. The alignment algorithm uses tracks that are reconstructed with the DAMPE offline software. It is performed with the cosmic data and relies on the minimisation of global $\chi^{2}$ of reconstructed tracks.
\end{abstract}

XXVII International Symposium on Lepton Photon Interactions at High Energies 17-22 August 2015

Ljubljana, Slovenia

\footnotetext{
${ }^{*}$ Speaker.

${ }^{\dagger}$ For the DAMPE collaboration
} 


\section{Introduction}

DAMPE (DArk Matter Particle Explorer) is a powerful satellite-borne telescope for high energy gamma-ray, electron and cosmic rays detection [1]. It consists of a double layer of plastic scintillator strips detector that serves as anti-coincidence detector (PSD), followed by silicon-tungsten tracker-converter (STK), which is made of 6 tracking double layers. Each layer consists of two layers of single-sided silicon strip detectors measuring the two orthogonal views perpendicular to the pointing direction of the apparatus. Three layers of tungsten plates with thickness of $1 \mathrm{~mm}$ each are inserted in front of tracking layer 2, 3 and 4 for photon conversion. The STK is followed by an imaging calorimeter of about 31 radiation lengths thickness, made up of 14 layers of Bismuth Germanium Oxide (BGO) bars in a hodoscopic arrangement. A layer of neutron detectors is added to the bottom of the calorimeter (NUD). A sketch of the detector is shown in Fig. 1. The total thickness of the BGO calorimeter and the STK makes DAMPE the deepest calorimeter ever used in space.
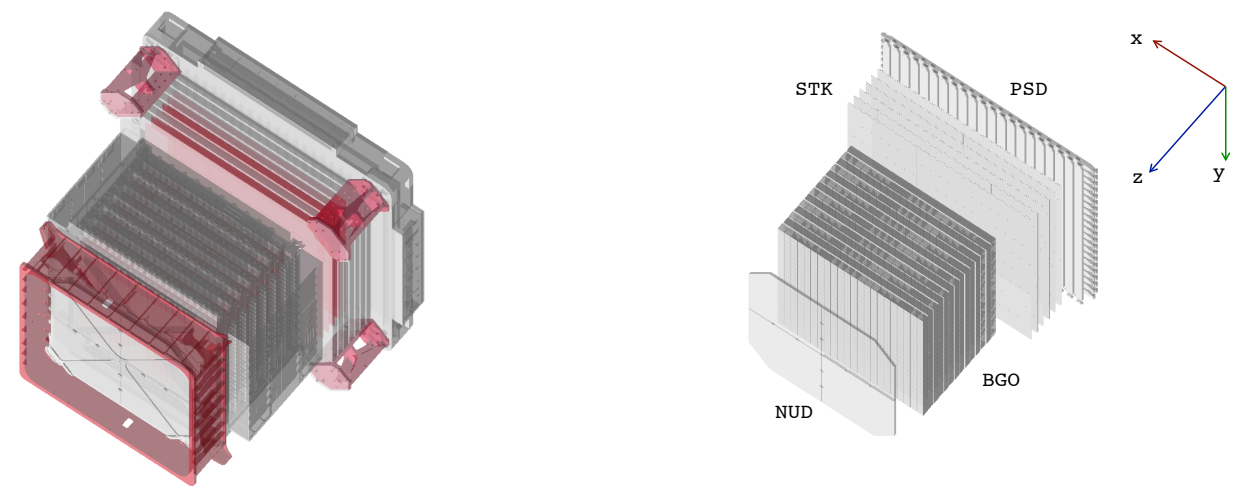

Figure 1: DAMPE as it appears in the GEANT4 viewer: left - whole satellite payload, right detector elements only (sensitive volumes).

In the proceeding we describe the implementation of conversion of CAD (Computer Aided Design) drawings of the DAMPE detector into the GDML format (Geometry Description Markup Language). The GDML is a geometry description standard utilised by GEANT4 [2] and other particle-physics simulation and reconstruction software. Furthermore, based on the offline software framework of DAMPE and its GDML geometry, preliminary alignment of the silicon tracker (STK) is performed. Detail description of the DAMPE software framework can be found in [3].

\section{DAMPE software framework and CAD to GDML conversion}

The framework of DAMPE offline software [3] is inspired by the GAUDI architecture [4]. The core part is implemented in $\mathrm{C}++$, where all the algorithms and services are inherited from the corresponding base classes. All computing jobs are configured in Python, in a so-called job-option files. The boost-python [5] libraries are used to interface algorithmic parts to the job-option scripts. The geometry of the detector in implemented in the GDML format from the direct conversion of the CAD drawings, whereby all the supporting structures of the instrument are taken into account in the simulation and reconstruction algorithms. There is no duplication of detector geometry, all parts 
of the software are working with the same geometry model. A dedicated service is implemented (in $\mathrm{C}++$ ) which allows to read a layout of sensitive parts (silicon sensors, BGO bars, etc.) of the detector from the GDML.

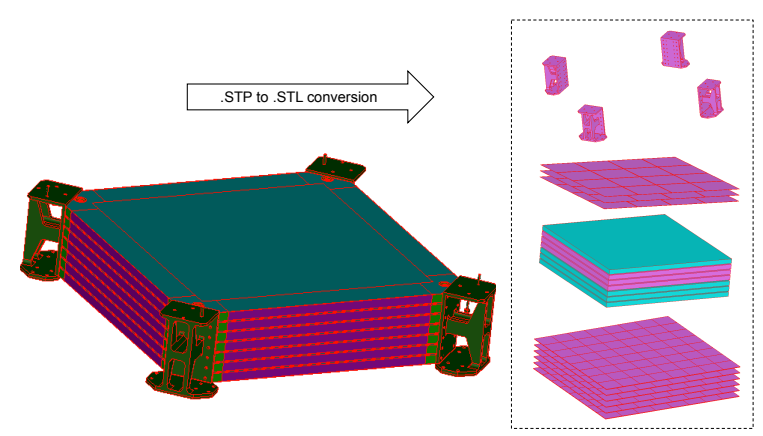

Figure 2: Conversion of CAD drawing of the STK into GDML format. The CAD model (.stp file) is exploded into a set of .stl files, each representing one material (silicons sensors, tungsten convertor plates, aluminium support structures, etc.)

It should be noted that the conversion of CAD drawings into GDML is a long-standing problem. The most widespread solution is based on conversion of CAD drawings into tessellated structures [6]. However, a straightforward conversion is not always possible, due to necessity of defining sensitive volumes. In case of STK, the number of read-out channels is 73728. Creating those as tessellated volumes would lead to enormous computational overhead both in simulation and reconstruction. In DAMPE we complement the above mentioned technique by replacing the sensitive parts of the detector (silicon sensors) with simple rectangular shapes. Silicon detectors are parsed from the geometry and split further into read-out strips. In doing so we bypass the problem of computational overhead of navigating through tessellated solids, while keeping the geometry exactly the same as in the provided CAD drawing. The CAD to GDML conversion is illustrated in Fig. 2. Before the conversion, an intermediate step is taken to explode the CAD model into parts, where each part corresponds to a single material. Next, those parts are converted into GDML modules, and proper materials are assigned to them. The conversion is done using a light-weight tool, running on any operating system with python in it, without installing additional libraries [7]. Finally, an XML file with the description of sensitive detectors (their positions) is created. The later one is used as an input to reconstruction jobs, providing mapping between read-out channels and $\mathrm{x}, \mathrm{y}, \mathrm{z}$ positions of the hits. The 3D model of DAMPE visualised with standard GEANT4 tools is shown in Fig. 1.

\section{Preliminary alignment of the silicon tracker of DAMPE}

Position resolution of silicon sensors of STK depends on the track impact angle and is as low as $40 \mu \mathrm{m}$ for the vertical angles [8]. in order to fully exploit this resolution, positions of silicon sensors in the STK must be evaluated (aligned) with precision of about $10 \mu \mathrm{m}$. The better is the alignment, the more precise is the angular resolution of STK. This is particularly important for the photon reconstruction. Preliminary alignment of the STK is done with the cosmic data collected 


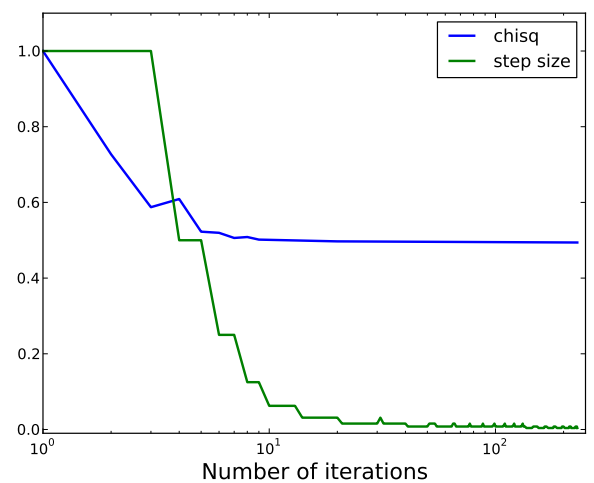

Figure 3: Normalised global $\chi^{2}$ and the length of the step in the phase space of the alignment parameters as a function of number of iterations of the alignment algorithm.

after the assembly of STK, in April 2015. The data set corresponds to one week of data-taking with the cosmic trigger, which yields about 6 Million events and about 1.5 Million tracks, which were reconstructed with the DAMPE software framework, and were refitted for the alignment with the straight lines using the least-squares method. All the tracks were required to have hits in all 6 layers of the STK (both $x$ and $y$ ). The global $\chi^{2}$ of the fit was evaluated:

$$
\chi^{2}=\sum_{i=1}^{N_{\text {tracks }}}\left(\sum_{k=1}^{6}\left(x_{i, k}^{f i t}-x_{i, k}^{\text {meas }}\right)^{2}+\sum_{k=1}^{6}\left(y_{i, k}^{f i t}-y_{i, k}^{\text {meas }}\right)^{2}\right)
$$

Alignment is performed by minimising the $\chi^{2}$ in the iterative procedure. At each iteration, derivatives of the $\chi^{2}$ are evaluated with respect to the alignment parameters, and a step is performed in the phase space of the alignment parameters according to the derivatives vector. The evolution of the $\chi^{2}$ as a function of iteration number is shown in Fig. 3. In the STK, the silicon sensors are mounted into so-called ladders. There are 192 ladders, each bearing 4 sensors [8]. Taking into account possible shifts ( 2 degrees of freedom) and rotations ( 3 degrees of freedom) of each silicon sensor with respect to the expected ideal position, this yields $192 \cdot 4 \cdot 5=3840$ alignment parameters to be evaluated. It should be noted that shifts of silicon sensors in the direction of the silicon strips are not sufficient, resulting in 2 translational degrees of freedom (not 3) per sensor. Initial positions (at 0-th step of the alignment) are taken from the GDML model. The alignment procedure terminates once the step in the phase space of the alignment parameters becomes smaller than a defined threshold.

A good indicators of the alignment quality are the track residue distributions. It is impossible to cover all those in this proceeding, hence we show only one of the characteristic examples. In Fig. 4 the track residue are shown before and after the alignment, as functions of track coordinates, for different STK layers. One can see that the spread of positions of silicon sensors in some cases reaches few hundred microns, while after the alignment the corresponding spread is within the range $\pm 10 \mu \mathrm{m}$. After the alignment, the global $\chi^{2}$ reduces almost twice, and the major convergence of the algorithm is reached within 10 steps (see Fig. 3). 

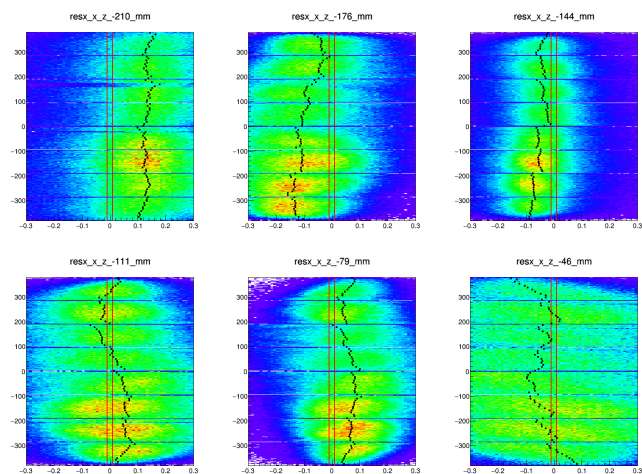
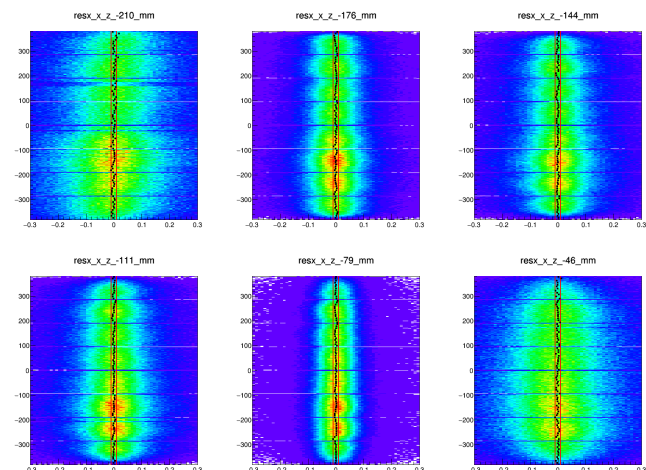

Figure 4: Residue of tracks with respect to measured track coordinates in the $x$-view of STK in 6 tracking layers: before (left) and after (right) the alignment; black dots are the mean values in the corresponding profiles of the histograms; $x$-axis ranges from -0.3 to $0.3 \mathrm{~mm}$ in all the plots; red lines are placed at -10 and $10 \mu \mathrm{m}$, to indicate the scale; top left plot corresponds to the tracking layer closest to the PSD, while bottom right is the one closest to the BGO.

\section{Conclusions}

The offline software of the DAMPE mission is discussed. An efficient method is implemented for converting CAD drawings of the detector into GDML model, which is used both by reconstruction and simulation jobs. Based on this software and geometry model, preliminary alignment of the silicon tracker of DAMPE is performed. After the alignment a spread of mean values of track residue throughout STK is well below twenty microns, much lower than intrinsic position resolution of silicon sensors.

\section{References}

[1] J. Chang, in The 7th international workshop " the Dark Side of the Universe" (DSU) 2011.

[2] S. Agostinelli et. al.,M Geant4 - a simulation toolkit, Nucl. Instrum. Meth. A 506, 3 (2003) 250. [http://dx.doi.org/10.1016/S0168-9002(03)01368-8].

[3] A. Tykhonov et. al., Software framework and the reconstruction software of the DAMPE gamma-ray mission, in proceeding of 34th ICRC, 1193 (2015).

[4] B. Hegner, P. Mato and D. Piparo, Evolving LHC data processing frameworks for efficient exploitation of new CPU architectures, [10.1109/NSSMIC . 2012.6551463].

[5] D. Abrahams, R. W. Grosse-Kunstleve, Building Hybrid Systems with Boost.Python, Boost Consulting, 2003,

[http://www.boost.org/doc/libs/1_31_0/libs/python/doc/PyConDC_2003/bpl.html].

[6] Christopher M Poole et. al., A CAD Interface for GEANT4, [phys ics . med-ph/1105. 0963].

[7] Andrii Tykhonov, CAD to GDML converter tool, [https://github.com/tihonav/cad-to-geant4-converter]

[8] V. Gallo et. al., The test results of the Silicon Tungsten Tracker of DAMPE, in proceeding of 34th ICRC, 1199 (2015). 